ELECTRONIC RESEARCH ANNOUNCEMENTS OF THE AMERICAN MATHEMATICAL SOCIETY

Volume 9, Pages 111-120 (November 13, 2003)

S $1079-6762(03) 00118-5$

\title{
NONHOLONOMIC TANGENT SPACES: INTRINSIC CONSTRUCTION AND RIGID DIMENSIONS
}

\author{
A. AGRACHEV AND A. MARIGO \\ (Communicated by Svetlana Katok)
}

\begin{abstract}
A nonholonomic space is a smooth manifold equipped with a bracket generating family of vector fields. Its infinitesimal version is a homogeneous space of a nilpotent Lie group endowed with a dilation which measures the anisotropy of the space. We give an intrinsic construction of these infinitesimal objects and classify all rigid (i.e. not deformable) cases.
\end{abstract}

\section{INTRODUCTION}

Let $M$ be a $\left(C^{\infty}\right)$ smooth connected $n$-dimensional manifold and $\mathcal{F} \subset$ Vec $M$ a set of smooth vector fields on $M$. Given $q \in M$ and an integer $l>0$ we set

$$
\Delta_{q}^{l}=\operatorname{span}\left\{\left[f_{1},\left[\ldots,\left[f_{i-1}, f_{i}\right] \cdots\right](q): f_{j} \in \mathcal{F}, 1 \leq j \leq i, i \leq l\right\} \subseteq T_{q} M .\right.
$$

Clearly, $\Delta_{q}^{l} \subseteq \Delta_{q}^{m}$ for $l<m$. The set $\mathcal{F}$ is called bracket generating (or completely nonholonomic) at $q$ if there exists $m_{q}$ such that $\Delta_{q}^{m_{q}}=T_{q} M$. The minimum among these $m_{q}$ is called the degree of nonholonomy of $\mathcal{F}$ at $q$. The set $\mathcal{F}$ is called bracket generating if it is bracket generating at every point.

We treat the pair $(M, \mathcal{F})$ as a "nonholonomic space", i.e. we assume that information propagates in $M$ only along the integral curves of fields from $\mathcal{F}$ and the concatenations of these curves. If $\mathcal{F}$ is bracket generating, then any point of $M$ is "reachable" from any other, i.e. points can be connected by an admissible curve. This is the Rashevskii-Chow theorem [5, 7]. Actually, one can derive more from this theorem. Let $t \mapsto e^{t f}$ be the (local) one-parameter subgroup of diffeomorphisms generated by a vector field $f \in \mathcal{F}$ so that $t \mapsto e^{t f}(q), q \in M$, are integral trajectories of $f$. The natural "nonholonomic topology" is the strongest topology on $M$ such that the mappings

$$
\left(t_{1}, \ldots, t_{k}\right) \mapsto e^{t_{1} f_{1}} \circ \ldots \circ e^{t_{k} f_{k}}(q), \quad f_{i} \in \mathcal{F} \quad q \in M, \quad k=1,2, \ldots,
$$

are continuous. It follows from the arguments of Rashevskii and Chow that this nonholonomic topology coincides with the standard topology on $M$.

Thus, the nonholonomic space $(M, \mathcal{F})$ is homeomorphic to the manifold $M$, but Analysis and Geometry on $(M, \mathcal{F})$ are very different from those on $M$. The main difference comes from the anisotropy of nonholonomic spaces: information propagates in all directions but with very different rates.

Received by the editors March 25, 2003.

2000 Mathematics Subject Classification. Primary 58A30; Secondary 58K50.

Key words and phrases. Nonholonomic system, nilpotent approximation, Carnot group. 
In this note we focus on tangent spaces to nonholonomic spaces. Let $q \in M$; we denote by $T_{q}^{\mathcal{F}} M$ the nonholonomic tangent space at $q$ to be defined. We should make some remarks before we go to its construction, since this kind of spaces, burdened by extraneous structures, already appeared in various contexts. First of all, the nonholonomic tangent functor is a generalization of the usual one: if $\mathcal{F}=\operatorname{Vec} M$ then $T_{q}^{\mathcal{F}} M$ coincides with $T_{q} M$. Actually the tangent space $T_{q}^{\mathcal{F}} M$ is homeomorphic to $\mathbb{R}^{n}$ for any bracket generating $\mathcal{F}$. Moreover, $T_{q}^{\mathcal{F}} M$ is a homogeneous space of a nilpotent Lie group, but in general, it has no intrinsic linear structure. On the other hand, it is intrinsically equipped with a canonical dilation which measures the anisotropy of the space.

Sub-Riemannian context. Assume that $\mathcal{F}$ is the space of sections of a vector subbundle of $T M$ (a vector distribution) and that $M$ is endowed with a Riemannian structure. The Carnot-Caratheodory distance between two points in $M$ is defined to be the infimum of all the lengths of admissible curves connecting these points. It is well known that the Carnot-Caratheodory metric spaces admit tangent metric spaces in the Gromov-Hausdorff sense. These tangent spaces are homeomorphic to $\mathbb{R}^{n}$ and their metrics are homogeneous with respect to certain dilations (see [3] for details). It turns out that the space and the dilation are defined independently on any metric and just represent $T_{q}^{\mathcal{F}} M$.

Other contexts are mathematical control theory and hypoelliptic operators. Nonholonomic tangent functors appear here via nilpotent or graded approximations of the anisotropic objects to be studied (see [1, 2, 4, 6, 8, 9, 10). The cited papers contain effective constructions and algorithms which allow one to compute things explicitly. A weak point of these constructions is their heavy dependence on the choice of coordinates. Because of that, the approximation looks like an auxiliary technical tool rather than a fundamental functorial operation; the geometric insight and the application of geometric machinery are highly impeded.

In this paper we introduce the nonholonomic tangent functor which performs the above-mentioned graded approximation in a natural coordinate-free way and demonstrates the intrinsic meaning of the dilation. One more goal of the paper is to classify all "rigid" cases. What does "rigid" mean? Obviously, $T_{q}^{\mathcal{F}} M$ depends only on the germ of $\mathcal{F}$ at $q$. Moreover, it depends only on the module generated by $\mathcal{F}$ over the algebra of germs at $q$ of smooth scalar functions. Assume that this module has $d$ generators $f_{1}, \ldots, f_{d}$; then $T_{q}^{\mathcal{F}} M=T_{q}^{\left\{f_{1} \ldots f_{d}\right\}} M$. We say that $T_{q}^{\left\{f_{1} \ldots f_{d}\right\}} M$ is rigid if $T_{q}^{\left\{f_{1} \ldots f_{d}\right\}} M$ is isomorphic to $T_{q}^{\left\{f_{1}^{\prime} \ldots f_{d}^{\prime}\right\}} M$ for all $f_{i}^{\prime}$ close enough to $f_{i}$ in the $C^{\infty}$ topology. The pair $(d, n)$ is called rigid bidimension if there exists at least one set of germs, $\left\{f_{1}, \ldots, f_{d}\right\} \subset \mathrm{Vec}_{0} \mathbb{R}^{n}$, such that $T_{0}^{\left\{f_{1} \ldots f_{d}\right\}} \mathbb{R}^{n}$ is rigid. Given a rigid bidimension $(d, n)$, it is not hard to show that a generic germ $\left\{f_{1}, \ldots, f_{d}\right\}$ is rigid and there is only a finite number of mutually nonisomorphic rigid $T_{0}^{\left\{f_{1} \ldots f_{d}\right\}} \mathbb{R}^{n}$.

In this paper we completely characterize the rigid bidimensions and, for each rigid bidimension, we indicate the number of different (up to isomorphism) $T_{0}^{\left\{f_{1} \ldots f_{d}\right\}} \mathbb{R}^{n}$. It turns out that this number can only be 1,2 , or 3 . Normal forms and proofs will be given in a forthcoming long paper.

\section{TANGENT FUnCTOR}

A flow in $M$ is a one-parameter family of diffeomorphisms $P_{t} \in$ Diff $M$ satisfying the condition $P_{0}=\mathrm{id}$. The set of flows is a group with respect to pointwise 
composition: the product of $P_{t}$ and $\hat{P}_{t}$ is the flow $t \mapsto P_{t} \circ \hat{P}_{t}$. Let $q \in M$. The tangent space $T_{q} M$ consists of 1 -jets at 0 of smooth curves $\gamma(t)$ in $M$ such that $\gamma(0)=q$. To construct the nonholonomic tangent space $T_{q}^{\mathcal{F}} M$ we need jets of order $m_{q}$, where $m_{q}$ is the degree of nonholonomy of $\mathcal{F}$ at $q$. The group of flows naturally acts on the space of smooth curves starting from $q$ : the flow $P_{t}$ sends the curve $\gamma(t)$ to the curve $P .(\gamma): t \mapsto P_{t}(\gamma(t))$. Of course, for any positive integer $l$, the $l$-jet at 0 of $P$. $(\gamma)$ depends only on $P_{t}$ and on the $l$-jet of $\gamma$. We thus obtain the action of the group of flows on the space $C_{q}^{l}$ of $l$-jets of smooth curves in $M$ starting from $q$. We keep the notation $q$ for the constant curve $\gamma(t) \equiv q$ and its jets; indeed, the trajectory $t \mapsto P_{t}(q)$ of the flow $P_{t}$ is the image of the constant curve under the natural action. The canonical dilation

$$
\delta_{\alpha}^{l}: C_{q}^{l} \rightarrow C_{q}^{l}, \quad \delta_{\alpha_{1} \alpha_{2}}^{l}=\delta_{\alpha_{1}}^{l} \circ \delta_{\alpha_{2}}^{l}, \quad \alpha \in \mathbb{R}, \quad \delta_{0}\left(C_{q}^{l}\right)=q,
$$

is induced by the standard dilation $\delta_{\alpha}$ on the space of smooth curves: $\left(\delta_{\alpha} \gamma\right)(t)=$ $\gamma(\alpha t)$ for any curve $\gamma$.

We denote by $p^{l, k}(f): C_{q}^{l} \rightarrow C_{q}^{l}$ the transformation induced by the flow $t \mapsto e^{t^{k}} f$, where $f \in \operatorname{Vec} M$. Let $\mathcal{P}^{l, \mathcal{F}}$ be the group of transformations generated by $\delta_{\alpha}^{l}$ with $\alpha \neq 0$, and by $p^{l, k}(f)$ with $f \in \mathcal{F}$ and $k=1,2,3, \ldots$ Dilations do not commute with flows, even at the jet level of $\mathcal{P}^{l, \mathcal{F}}$. However, given a flow $P_{t}$, one can see that $\lim _{s \rightarrow 0}\left(\delta_{s}^{l} \circ P . \circ \delta_{s^{-1}}^{l}\right)=$ id on the space of curves. This motivates the definition $D(\phi)=\lim _{s \rightarrow 0}\left(\delta_{s}^{l} \circ \phi \circ \delta_{s^{-1}}^{l}\right), \phi \in \mathcal{P}^{l, \mathcal{F}}$. Then the image of the group homomorphism $D$ is the dilation group $\left\{\delta_{\alpha}: \alpha \neq 0\right\}$.

We denote by $\mathcal{O}^{l, \mathcal{F}} \subset C_{q}^{l}$ the orbit of the jet $q$ under the action of the group $\mathcal{P}^{l, \mathcal{F}}$. Let us also consider the normal subgroup $\mathcal{P}_{\circ}^{l, \mathcal{F}}$ of $\mathcal{P}^{l, \mathcal{F}}$ generated by $p^{l, k}(f)$, $f \in \mathcal{F}, k=2,3, \ldots$ (the difference from $\mathcal{P}^{l, \mathcal{F}}$ is that $p^{l, 1}(f)$ and $\delta_{\alpha}^{l}$ are absent). Finally, we set:

- $T_{q}^{l, \mathcal{F}} M$, the quotient space of $\mathcal{O}^{l, \mathcal{F}}$ by the action of the group $\mathcal{P}_{\circ}^{l, \mathcal{F}}$;

- $T_{q} \mathcal{P}^{l, \mathcal{F}}$, the group of transformations of $T_{q}^{l, \mathcal{F}} M$ induced by the action of $\mathcal{P}^{l, \mathcal{F}} / \mathcal{P}_{\circ}^{l, \mathcal{F}}$

We keep the symbols $\delta_{\alpha}^{l}$ and $p^{l, k}(f)$ for the induced transformations of $T_{q}^{l, \mathcal{F}} M$, and the symbol $D$ for the induced homomorphism of $T_{q} \mathcal{P}^{l, \mathcal{F}}$ on $\left\{\delta_{\alpha}: \alpha \neq 0\right\}$. See Section 3 for further understanding of these objects.

Proposition 2.1. The codimension 1 normal subgroup $D^{-1}(\mathrm{id}) \subset T_{q} \mathcal{P}^{l, \mathcal{F}}$ is a nilpotent Lie group generated by the one-parameter subgroups $s \mapsto p^{l, 1}(s f), f \in \mathcal{F}$. The group $D^{-1}(\mathrm{id})$ acts transitively on $T_{q}^{l, \mathcal{F}} M$.

Let $\pi^{l}: C_{q}^{l} \rightarrow C_{q}^{l-1}$ be the standard projection. Obviously, $\pi^{l} \circ p^{l, k}(f)=$ $p^{l-1, k}(f) \circ \pi^{l}, \quad \pi^{l} \circ \delta_{\alpha}^{l}=\delta_{\alpha}^{l-1} \circ \pi^{l}$. Hence $\pi^{l}$ sends orbits of the groups $\mathcal{P}^{l, \mathcal{F}}$ and $\mathcal{P}_{\circ}^{l, \mathcal{F}}$ to orbits of the groups $\mathcal{P}^{l-1, \mathcal{F}}$ and $\mathcal{P}_{\circ}^{l-1, \mathcal{F}}$ and induces a mapping on the quotient spaces. We keep the symbol $\pi^{l}$ for the induced mapping so that $\pi^{l}: T_{q}^{l, \mathcal{F}} M \rightarrow T_{q}^{l-1, \mathcal{F}} M$.

Proposition 2.2. For any $l>0, \pi^{l}: T_{q}^{l, \mathcal{F}} M \rightarrow T_{q}^{l-1, \mathcal{F}} M$ is a fiber bundle with fiber $\Delta_{q}^{l} / \Delta_{q}^{l-1}$.

In particular, $T_{q}^{l, \mathcal{F}} M$ is diffeomorphic to $\Delta_{q}^{l}$ and $T_{q}^{l, \mathcal{F}} M=T_{q}^{m_{q}, \mathcal{F}} M$ for $l \geq m_{q}$, where $m_{q}$ is the degree of nonholonomy. Moreover, one can show that $T_{q} \mathcal{P}^{l, \mathcal{F}}=$ $T_{q} \mathcal{P}^{m_{q}, \mathcal{F}}$ for $l \geq m_{q}$ as well. 
Definition. Let $l$ be greater than or equal to the degree of nonholonomy, i.e. $\Delta_{q}^{l}=$ $T_{q} M$. The nonholonomic tangent space $T_{q}^{\mathcal{F}} M$ is the manifold $T_{q}^{l, \mathcal{F}} M$ equipped with the transitive action of the group $T_{q} \mathcal{P}^{\mathcal{F}} \stackrel{\text { def }}{=} T_{q} \mathcal{P}^{l, \mathcal{F}}$. For any $f \in \mathcal{F}$, the vector field $T_{q}^{\mathcal{F}} f \in \operatorname{Vec} T_{q}^{\mathcal{F}} M$ is the generator of the one-parameter group $s \mapsto p^{l, 1}(s f)$; in other words, $e^{s T_{q}^{\mathcal{F}} f \stackrel{\text { def }}{=}} p^{l, 1}(s f)$.

Obviously, $f \mapsto T_{q}^{\mathcal{F}} f$ is a homomorphism of Lie algebras of vector fields; the group $T_{q} \mathcal{P}^{\mathcal{F}}$ is generated by the dilation and the one-parameter subgroups $e^{s T_{q}^{\mathcal{F}} f}$, $s \in \mathbb{R}, f \in \mathcal{F}$. Moreover, just from the fact that the definition of $T_{q}^{\mathcal{F}} M$ is intrinsic, it follows that every diffeomorphism $\Phi: M \rightarrow M$ automatically induces an equivariant mapping $\Phi_{*}^{\mathcal{F}}: T_{q}^{\mathcal{F}} M \rightarrow T_{\Phi(q)}^{\Phi_{*} \mathcal{F}} M$ such that $\left(\Phi_{1} \circ \Phi_{2}\right)_{*}^{\mathcal{F}}=\Phi_{1 *}^{\Phi_{2 *} \mathcal{F}} \circ \Phi_{2 *}^{\mathcal{F}}$ for any pair of diffeomorphisms $\Phi_{1}, \Phi_{2}$. One more functorial property is as follows. Assume that $\mathcal{F} \subset \mathcal{G} \subset$ Vec $M$; the identity inclusion $\imath: \mathcal{F} \rightarrow \mathcal{G}$ induces a homomorphism $\imath_{*}: T_{q} \mathcal{P}^{\mathcal{F}} \rightarrow T_{q} \mathcal{P}^{\mathcal{G}}$ and an equivariant smooth mapping $\imath_{*}: T_{q}^{\mathcal{F}} M \rightarrow T_{q}^{\mathcal{G}} M$.

Proposition 2.3. Let $\overline{\mathcal{F}}=\left\{\sum_{j=1}^{k} a_{j} f_{j}: f_{j} \in \mathcal{F}, a_{j} \in C^{\infty}(M), k>0\right\}$ be the module over $C^{\infty}(M)$ generated by $\mathcal{F}$, and let $\imath: \mathcal{F} \rightarrow \overline{\mathcal{F}}$ be the identity inclusion. Then $\imath_{*}:\left(T_{q} \mathcal{P}^{\mathcal{F}}, T_{q}^{\mathcal{F}} M\right) \longrightarrow\left(T_{q} \mathcal{P}^{\overline{\mathcal{F}}}, T_{q}^{\overline{\mathcal{F}}} M\right)$ is an isomorphism.

Remark. The last proposition states that nonholonomic tangent spaces depend on the submodule of Vec $M$ generated by $\mathcal{F}$ rather than on $\mathcal{F}$ itself. In fact, from the very beginning of the paper, we could deal with submodules of Vec $M$ instead of subsets. This approach would provide slightly more general functorial properties, but the whole construction would become even more dry and abstract than it is now. Anyway, the algebraically trained reader will easily recover the missing functorial properties.

\section{Coordinate presentation}

Given nonnegative integers $k_{1}, \ldots, k_{l}$, where $k_{1}+\cdots+k_{l}=n$, we present $\mathbb{R}^{n}$ as the direct sum $\mathbb{R}^{k_{1}} \oplus \cdots \oplus \mathbb{R}^{k_{l}}$. Every vector $x \in \mathbb{R}^{n}$ can be written as

$$
x=\left(x_{1}, \ldots, x_{l}\right), \quad x_{i}=\left(x_{i 1}, \ldots, x_{i k_{i}}\right) \in \mathbb{R}^{k_{i}}, i=1, \ldots, l .
$$

A differential operator on $\mathbb{R}^{n}$ with smooth coefficients has the form $\sum_{\alpha} a_{\alpha}(x) \frac{\partial^{|\alpha|}}{\partial x^{\alpha}}$, where $a_{\alpha} \in C^{\infty}\left(\mathbb{R}^{n}\right)$ and $\alpha$ is a multiindex: $\alpha=\left(\alpha_{1}, \ldots, \alpha_{l}\right), \alpha_{i}=\left(\alpha_{i 1}, \ldots, \alpha_{i k_{i}}\right)$, $\left|\alpha_{i}\right|=\sum_{j=1}^{k_{i}} \alpha_{i j}, i=1, \ldots, l$. The space of all differential operators with smooth coefficients is an associative algebra with composition of operators as multiplication. The set of differential operators with polynomial coefficients is a subalgebra of this algebra with generators $1, x_{i j}, \frac{\partial}{\partial x_{i j}}, i=1, \ldots, l, j=1, \ldots, k_{i}$. We introduce a $\mathbb{Z}$-grading of this subalgebra by assigning weights $\nu$ to the generators: $\nu(1)=0$, $\nu\left(x_{i j}\right)=i$, and $\nu\left(\frac{\partial}{\partial x_{i j}}\right)=-i$. Accordingly

$$
\nu\left(x^{\alpha} \frac{\partial^{|\beta|}}{\partial x^{\beta}}\right)=\sum_{i=1}^{l}\left(\left|\alpha_{i}\right|-\left|\beta_{i}\right|\right) i,
$$

where $\alpha$ and $\beta$ are multiindices.

A differential operator with polynomial coefficients is said to be $\nu$-homogeneous of weight $m$ if all monomials occurring in it have weight $m$. It is easy to see that $\nu\left(A_{1} \circ A_{2}\right)=\nu\left(A_{1}\right)+\nu\left(A_{2}\right)$ for any $\nu$-homogeneous differential operators $A_{1}$ and $A_{2}$. 
The most important for us are the differential operators of order 0 (functions) and of order 1 (vector fields). We have $\nu(g a)=\nu(g)+\nu(a), \nu\left(\left[g_{1}, g_{2}\right]\right)=\nu\left(g_{1}\right)+\nu\left(g_{2}\right)$ for any $\nu$-homogeneous function $a$ and vector fields $g, g_{1}, g_{2}$. A differential operator of order $N$ has weight at least $-N l$; in particular, the weight of a nonzero vector field is at least $-l$. Vector fields of nonnegative weights vanish at 0 while the values at 0 of fields of weight $-i$ belong to the subspace $\mathbb{R}^{k_{i}}$, the $i$ th term in the presentation $\mathbb{R}^{n}=\mathbb{R}^{k_{1}} \oplus \cdots \oplus \mathbb{R}^{k_{l}}$.

We introduce a dilation $\delta_{t}: \mathbb{R}^{n} \rightarrow \mathbb{R}^{n}, t>0$, by setting

$$
\delta_{t}\left(x_{1}, x_{2}, \ldots, x_{l}\right)=\left(t x_{1}, t^{2} x_{2}, \ldots, t^{l} x_{l}\right)
$$

The $\nu$-homogeneity means homogeneity with respect to this dilation. In particular, we have that $a\left(\delta_{t} x\right)=t^{\nu(a)} a(x), \delta_{t *} g=t^{-\nu(g)} g$ for a $\nu$-homogeneous function $a$ and a vector field $g$.

Now let $g=\sum_{i, j} a_{i j} \frac{\partial}{\partial x_{i j}}$ be an arbitrary smooth vector field. Expanding the coefficients $a_{i j}$ in a Taylor series in powers of $x_{i j}$ and grouping terms with the same weights, we get an expansion $g \approx \sum_{m=-l}^{+\infty} g^{(m)}$, where $g^{(m)}$ is a $\nu$-homogeneous field of weight $m$. This expansion enables us to introduce a decreasing filtration in the Lie algebra of smooth vector fields $\operatorname{Vec} \mathbb{R}^{n}$ by putting

$$
\operatorname{Vec}^{m}\left(k_{1}, \ldots, k_{l}\right)=\left\{X \in \operatorname{Vec} \mathbb{R}^{n}: X^{(i)}=0 \text { for } i<m\right\}, \quad-l \leq m<+\infty .
$$

It is easy to see that

$$
\left[\operatorname{Vec}^{m_{1}}\left(k_{1}, \ldots, k_{l}\right), \operatorname{Vec}^{m_{2}}\left(k_{1}, \ldots, k_{l}\right)\right] \subseteq \operatorname{Vec}^{m_{1}+m_{2}}\left(k_{1}, \ldots, k_{l}\right) .
$$

It happens that this class of filtrations is in a sense universal. The following theorem is a special case of the general results proved in [2, 4.

Theorem 3.1. Suppose that $\operatorname{dim}\left(\Delta_{q}^{i} / \Delta_{q}^{i-1}\right)=k_{i}, i=1, \ldots, l$. Then there exists a neighborhood $O_{q}$ of $q$ in $M$ and a coordinate mapping $\chi: O_{q} \rightarrow \mathbb{R}^{n}$ such that

$$
\chi(q)=0, \quad \chi_{*}\left(\Delta_{q}^{i}\right)=\mathbb{R}^{k_{1}} \oplus \cdots \oplus \mathbb{R}^{k_{i}}, 1 \leq i \leq l,
$$

and $\chi_{*}(\mathcal{F}) \subset \operatorname{Vec}^{-1}\left(k_{1}, \ldots, k_{l}\right)$.

A mapping $\chi: O_{q} \rightarrow \mathbb{R}^{n}$ which satisfies the hypothesis of the above theorem is called an adapted coordinate map for $\mathcal{F}$. All constructions have a simple explicit presentation in adapted coordinates. Unfortunately, an adapted coordinate map for a given $\mathcal{F}$ is far from being unique and it is not clear how to select a canonical one.

In any coordinates, the $l$-jet of a curve is identified with its degree $l$ Taylor polynomial. In particular, $\chi_{*}\left(C_{q}^{l}\right)=\left\{\sum_{i=1}^{l} t^{i} \xi_{i}: \xi_{i} \in \mathbb{R}^{n}\right\}$. 
Proposition 3.2. Let $\chi$ be an adapted coordinate map for $\mathcal{F}$; then

$$
\chi_{*}\left(\mathcal{O}^{l, \mathcal{F}}\right)=\left\{\sum_{i=1}^{l} t^{i} \xi_{i}: \xi_{i} \in \mathbb{R}^{k_{1}} \oplus \cdots \oplus \mathbb{R}^{k_{i}}, 1 \leq i \leq l\right\}
$$

and, for any $\gamma \in \mathcal{O}^{l, \mathcal{F}}$,

$$
\chi_{*}\left(\mathcal{P}_{\circ}^{l, \mathcal{F}}(\gamma)\right)=\chi_{*}(\gamma)+\left\{\sum_{i=1}^{l} t^{i} \xi_{i}: \xi_{i} \in \mathbb{R}^{k_{1}} \oplus \cdots \oplus \mathbb{R}^{k_{i-1}}, 1 \leq i \leq l\right\} .
$$

The proof of this proposition is based on the expansions

$$
\begin{aligned}
& \chi_{*}(f) \approx \chi_{*}(f)^{(-1)}+\sum_{j=0}^{\infty} \chi_{*}(f)^{(j)} \quad \forall f \in \mathcal{F}, \\
& \chi\left(e^{\tau f}(q)\right) \approx \sum_{i=1}^{\infty} \frac{\tau^{i}}{i !} \underbrace{\left(\chi_{*} f\right) \circ \cdots \circ\left(\chi_{*} f\right)}_{i \text { times }} I d(0),
\end{aligned}
$$

where $\operatorname{Id}(x) \equiv x, x \in \mathbb{R}^{n}$, and on the fact that all $\nu$-homogeneous functions of positive weight vanish at 0 .

Proposition 2.3 implies the identification of $T_{0}^{\chi_{*}(\mathcal{F})} \mathbb{R}^{n}$ with $\left\{\sum_{i=1}^{l} t^{i} \xi_{i}: \xi_{i} \in \mathbb{R}^{k_{i}}\right.$, $1 \leq i \leq l\}$. Similarly, the vector field $T_{0}^{\chi_{*}(\mathcal{F})} f$ is identified with $t\left(\chi_{*}(f)\right)^{(-1)}$. In particular, the fields $T_{q}^{\mathcal{F}} f, f \in \mathcal{F}$, are represented by $\nu$-homogeneous vector fields of weight -1 in adapted coordinates.

\section{Regularity AND RIGIDITY}

In general, the nilpotent Lie algebra generated by the fields $T_{q}^{\mathcal{F}} f, f \in \mathcal{F}$, may have a dimension strictly greater than $\operatorname{dim} T_{q}^{\mathcal{F}} M=\operatorname{dim} M$, and $T_{q}^{\mathcal{F}} M$ may not have an intrinsic Lie group structure. A simple example is provided by the Martinet distribution $\mathcal{F}=\operatorname{span}\left\{\frac{\partial}{\partial x_{1}}, \frac{\partial}{\partial x_{2}}+x_{1}^{2} \frac{\partial}{\partial x_{3}}\right\}$ in $\mathbb{R}^{3}$, where $T_{q}^{\mathcal{F}} f, f \in \mathcal{F}$, generate a 4-dimensional Lie algebra. This phenomenon does not occur in the so-called regular case, first studied by Vershik and Gershkovich (see [8, 9, 10]).

Definition. We say that $\mathcal{F} \subset \operatorname{Vec} M$ is regular at $q_{0} \in M$ if $\operatorname{dim} \Delta_{q}^{i}$ is constant in a neighborhood of $q_{0}, \forall i>0$.

Let $\mathcal{F}$ be regular at $q$ and $\operatorname{dim} \Delta_{q}^{1}=d$. Take $f_{1}, \ldots, f_{d} \in \mathcal{F}$ such that the vectors $f_{1}\left(q_{0}\right), \ldots, f_{d}\left(q_{0}\right)$ form a basis of $\Delta_{q_{0}}^{1}$. Then $f_{1}(q), \ldots, f_{d}(q)$ form a basis of $\Delta_{q}^{1}$ for any $q$ in a neighborhood of $q_{0}$. Hence, for any $f \in \mathcal{F}$ there exist smooth functions $a_{1}, \ldots, a_{d}$ such that $f(q)=\sum_{i=1}^{d} a_{i}(q) f_{i}(q)$ for any $q$ in the same neighborhood. It follows that

$$
\Delta_{q}^{l}=\operatorname{span}\left\{\left[f_{i_{1}},\left[\ldots, f_{i_{l}}\right] \cdots\right](q): 1 \leq i_{j} \leq d\right\}+\Delta_{q}^{l-1}, \quad l=1,2, \ldots .
$$

By the regularity of $\mathcal{F}$ at $q$, one can select vector fields from the collection $\left\{\left[f_{i_{1}},\left[\ldots, f_{i_{l}}\right] \cdots\right](q): 1 \leq i_{j} \leq d\right\}$ in such a way that their values at $q$ form a basis of $\Delta_{q}^{l} / \Delta_{q}^{l-1}$ for all $q$ close enough to $q_{0}$. With these bases in hand we easily obtain the following well-known fact: 
Lemma 4.1. Assume that $\mathcal{F} \subset \operatorname{Vec} M$ is regular at $q_{0}, v_{i}, v_{j} \in \operatorname{Vec} M, v_{i}(q) \in$ $\Delta_{q}^{i}, v_{j}(q) \in \Delta_{q}^{j} \forall q$, and $v_{i}\left(q_{0}\right)=0$. Then $\left[v_{i}, v_{j}\right]\left(q_{0}\right) \in \Delta_{q_{0}}^{i+j-1}$.

It follows immediately from this lemma that the Lie brackets of vector fields with values in $\Delta_{q}^{i}, i=1,2, \ldots$, induce a structure of graded Lie algebra on the space $\sum_{i>0} \Delta_{q_{0}}^{i} / \Delta_{q_{0}}^{i-1}$. We denote this graded Lie algebra by $\operatorname{Lie}_{q_{0}} \mathcal{F}$. Obviously, $\operatorname{Lie}_{q_{0}} \mathcal{F}$ is generated by $\Delta_{q_{0}}^{1}$.

Proposition 4.2. Let $\mathcal{F}$ be regular and bracket generating at $q \in M$. Then the mapping $f \mapsto T_{q}^{\mathcal{F}} f, f \in \mathcal{F}$, induces a Lie algebra isomorphism of $\operatorname{Lie}_{q} \mathcal{F}$ and $\operatorname{Lie}\left\{T_{q}^{\mathcal{F}} f: f \in \mathcal{F}\right\}$.

Recall that $\operatorname{Lie}\left\{T_{q}^{\mathcal{F}} f: f \in \mathcal{F}\right\}$ is the Lie algebra of a codimension 1 normal subgroup of $T_{q} \mathcal{P}^{\mathcal{F}}$, which acts transitively on $T_{q}^{\mathcal{F}} M$ (see Proposition [2.1). The quotient of $T_{q} \mathcal{P}^{\mathcal{F}}$ by this subgroup is the dilation. We thus have a transitive action of the $n$-dimensional nilpotent Lie group generated by the Lie algebra $\operatorname{Lie}_{q} \mathcal{F}$ on $T_{q} M$. Since $T_{q}^{\mathcal{F}} M$ is diffeomorphic to $\mathbb{R}^{n}$ and has the "origin" (the jet of the constant curve $q$ ), we obtain a canonical isomorphism of $T_{q}^{\mathcal{F}} M$ with the simply connected Lie group generated by $\operatorname{Lie}_{q} \mathcal{F}$.

We now turn to the generic case. Let $\mathcal{L}_{d}$ be the free Lie algebra with $d$ generators (all algebras in this paper are over $\mathbb{R}$ ); in other words, $\mathcal{L}_{d}$ is the Lie algebra of commutator polynomials of $d$ variables. We have $\mathcal{L}_{d}=\bigoplus_{i=1}^{\infty} \mathcal{L}_{d}^{i}$, where $\mathcal{L}_{d}^{i}$ is the space of degree $i$ homogeneous commutator polynomials. We set $\ell_{d}(i)=\operatorname{dim} \mathcal{L}_{d}^{i}, \ell_{d}^{(i)}=$ $\sum_{j=1}^{i} \ell_{d}(i)$. The classical recursion expression of $\ell_{d}(i)$ is $\ell_{d}(i)=d^{i}-\sum_{j \mid i} j \ell_{d}(j)$.

Below we deal with the space of germs at $q \in M$ of $d$-tuples of smooth vector fields $\left(f_{1}, \ldots, f_{d}\right)$ endowed with the standard $C^{\infty}$ topology. The following statement is almost obvious.

Proposition 4.3. For an open, everywhere dense set of d-tuples $\left(f_{1}, \ldots, f_{d}\right)$, the set $\mathcal{F}=\left\{f_{1}, \ldots, f_{d}\right\}$ is regular and bracket generating at $q$ with degree of nonholonomy $m_{q}=\min \left\{i: \ell_{d}^{(i)} \geq n\right\}$ and $\operatorname{dim}\left(\Delta_{q}^{i} / \Delta_{q}^{i-1}\right)=\ell_{d}(i)$ for $i=1, \ldots, m_{q}-1$.

Take $\mathcal{F}$ such that the module $\overline{\mathcal{F}}$ is generated by a generic $d$-tuple of vector fields. According to Propositions 4.2 and 4.3 the classification of $\left(T_{q} \mathcal{P}^{\mathcal{F}}, T_{q}^{\mathcal{F}} M\right)$ for such $\mathcal{F}$ is reduced to the classification of generic graded Lie algebras $\operatorname{Lie}_{q} \mathcal{F}$ or corresponding Lie groups.

Definitions of rigidity and of rigid bidimensions were given in the Introduction (isomorphism in this case means just Lie group isomorphism). In the next theorem we list all rigid bidimensions. It is convenient to give special names to some infinite series of bidimensions. For $d=2,3,4, \ldots$, the bidimensions $\left(d, \ell_{d}^{(i)}\right), i=1,2,3, \ldots$, are called free; the bidimension $(d, d+1)$ is called the Darboux bidimension, and the bidimension $(d,(d-1)(d+2) / 2)$ is called the dual Darboux bidimension. 
Theorem 4.4. All free, Darboux, and dual Darboux bidimensions are rigid; each of these bidimensions admits a unique up to isomorphism rigid group. Besides, there are 16 exceptional rigid bidimensions:

$(2,4)_{1},(2,6)_{2},(2,7)_{2},(4,6)_{2},(4,7)_{2},(4,8)_{2}$,

$(5,7)_{1},(5,8)_{2},(5,9)_{3},(5,11)_{3},(5,12)_{2},(5,13)_{1}$,

$(6,8)_{2},(6,19)_{2},(7,9)_{1},(7,26)_{1}$,

where the index $j$ in the expression $(d, n)_{j}$ indicates the number of isomorphism classes of rigid groups for the bidimension $(d, n)$.

There are no other rigid bidimensions.

Remark. This theorem is based on a complete classification of rigid groups, which will be published in a separate paper. An interesting open problem is to classify all "simple" $T_{q}^{\left\{f_{1}, \ldots, f_{d}\right\}} M$. The term comes from singularity theory: a nonholonomic tangent space $T_{q}^{\left\{f_{1}, \ldots, f_{d}\right\}} M$ is called simple if its small perturbations $T_{q}^{\left\{f_{1}^{\prime}, \ldots, f_{d}^{\prime}\right\}} M$ admit only a finite number of isomorphism classes. The above-mentioned Martinet distribution is an example of a nonrigid simple case. Obviously, all simple cases must live in rigid bidimensions.

\section{Generalization}

The construction of the functor $T_{q}^{\mathcal{F}}$ is easily generalized to the case of an $\mathcal{F}$ with a fixed filtration (when various fields from $\mathcal{F}$ have various "weights"). More precisely, let

$$
\mathcal{F}_{0} \subseteq \mathcal{F}_{1} \subseteq \cdots \subseteq \mathcal{F}_{\nu} \subseteq \cdots=\mathcal{F}
$$

and $f_{0}(q)=0$ for any $f_{0} \in \mathcal{F}_{0}$. Then the (local) diffeomorphism $e^{f_{0}}$ induces a transformation $p^{l, 0}(f)$ of $C_{q}^{l}$. We now set:

- $\Delta_{q}^{l}=\operatorname{span}\left\{\left[f_{1},\left[\ldots,\left[f_{i-1}, f_{i}\right] \cdots\right](q): f_{j} \in \mathcal{F}_{\nu_{j}}, \nu_{1}+\cdots+\nu_{i} \leq l, i>0\right\}\right.$

- $\mathcal{P}^{l}(\mathcal{F})$, the group of transformations generated by $\delta_{\alpha}^{l}, \alpha \in \mathbb{R} \backslash 0$ and by $p^{l, k}(f), f \in \mathcal{F}_{k}, k \geq 0$

- $\mathcal{P}_{\circ}^{l}(\mathcal{F})$, the normal subgroup of $\mathcal{P}^{l}(\mathcal{F})$ generated by $p^{l, k}(f), f \in \mathcal{F}_{k-1}$, $k>0$;

and repeat the whole construction with these modified definitions. The presence of $\mathcal{F}_{0}$ brings some new phenomena. In particular, the Lie algebra generated by $T_{q} f$, $f \in \mathcal{F}$, is not, in general, nilpotent. Moreover, the substitution for $\mathcal{F}$ of the module $\overline{\mathcal{F}}$ with the induced filtration may enlarge this Lie algebra.

Typical examples are smooth nonlinear control systems with an equilibrium at $(q, 0)$ :

$$
\dot{x}=f(x, u), \quad x \in M, u \in \mathbb{R}^{r}, \quad f(q, 0)=0 .
$$

We set $\mathcal{F}_{\nu}=\left\{\frac{\partial^{|\alpha|}}{\partial u^{\alpha}} f(\cdot, 0):|\alpha| \leq \nu\right\}$; the induced filtration of the module $\overline{\mathcal{F}}$ is feedback invariant. If the linearization of system $(2)$ at $(q, 0)$ is controllable, then the tangent functor provides exactly the linearization and its module version admits a finite classification (Brunovsky normal forms). The number of isomorphism classes equals the number of partitions of $r$. It would be very interesting to study the tangent functor for some classes of systems with noncontrollable linearizations. 


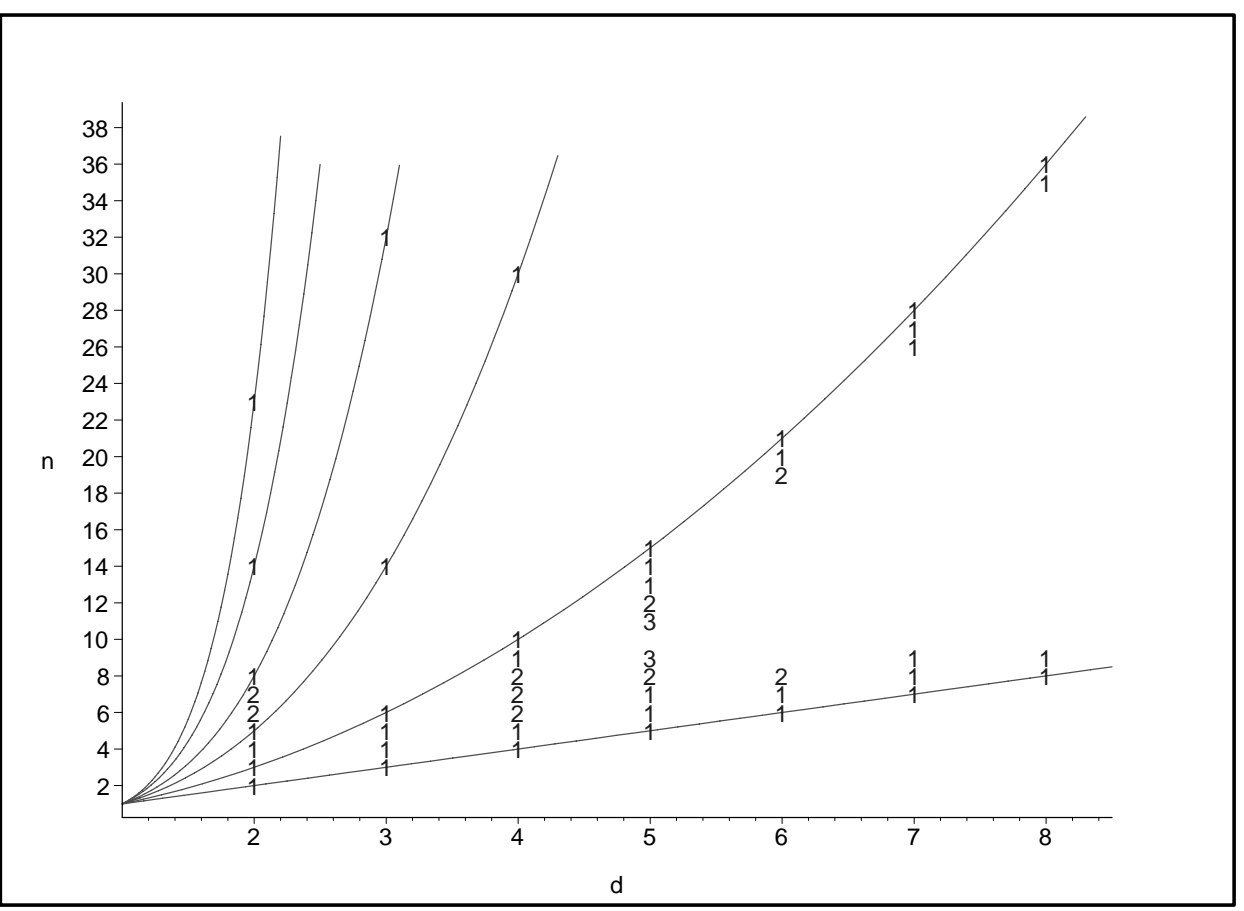

FIGURE 1. Rigid bidimensions with the indication of the number of isomorphism classes. The free bidimensions lie on the curves.

\section{REFERENCES}

[1] A. A. Agrachev, A. V. Sarychev, Filtrations of a Lie algebra of vector fields and nilpotent approximation of control systems. Dokl. Akad. Nauk SSSR 295 (1987); English transl., Soviet Math. Dokl. 36 (1988), 104-108. MR 88j:93015

[2] A. A. Agrachev, R. V. Gamkrelidze, A. V. Sarychev, Local invariants of smooth control systems. Acta Appl. Math. 14 (1989), 191-237. MR 90i:93033

[3] A. Bellaiche, The tangent space in sub-Riemannian geometry. In: Sub-Riemannian geometry, Birkhäuser, Progress in Math. 144 (1996), 1-78. MR 98a:53108

[4] R. M. Bianchini, G. Stefani, Graded approximations and controllability along a trajectory. SIAM J. Control Optim. 28 (1990), 903-924. MR 91d:93006

[5] W-L. Chow, Über Systeme von linearen partiellen Differentialgleichungen ester Ordnung. Math. Ann. 117 (1940/41), 98-105. MR 1:313d

[6] L. M. Rothschild, E. M. Stein, Hypoelliptic differential operators and nilpotent groups. Acta Math. 137 (1976), 247-320. MR 55:9171

[7] P. K. Rashevskii, About connecting two points of a completely nonholonomic space by an admissible curve. Uch. Zapiski Ped. Inst. Libknechta, No. 2 (1938), 83-94. (Russian)

[8] A. M. Vershik, V. Ya. Gershkovich, Nonholonomic dynamic systems. Geometry of distributions and variational problems. Springer Verlag, EMS 16 (1987), 5-85. MR 89f:58007 
[9] A. M. Vershik, V. Ya. Gershkovich, A bundle of nilpotent Lie algebras over a nonholonomic manifold (nilpotenization). Zap. Nauch. Sem. LOMI 172 (1989), 3-20. English transl., J. Soviet Math. 59 (1992), 1040-1053. MR 91a:58012

[10] A. M. Vershik, V. Ya. Gershkovich, Estimation of the functional dimension of the orbit space of germs of distributions in general position. Mat. Zametki 44 (1988), no. 5, 596-603, 700. English transl., Math. Notes 44 (1988), no. 5-6, 806-810 (1989) MR 90g:58002

Steklov Mathematical Institute, Moscow, Russia Current address: SISSA, Via Beirut 2-4, Trieste, Italy

E-mail address: agrachev@ma.sissa.it

iaC-CNR, Viale Policlinico 136, Roma, Italy

E-mail address: marigo@iac.rm.cnr.it 